trogones describe bends and sinuosities of a most peculiar character. As Dr. Naumann states, these results seem to open up a new field of research quite worth investigating.

A PAPER of unusual scientific interest was read at Monday's meeting of the Royal Geographical Society by Mr. H. J. Mackinder, B.A. (Oxford), on the field and methods of geography. Mr. Mackinder aimed at showing how geography could be made more than a mere cultivation of dry facts, and become indeed a department of scientific inquiry. He takes man as the centre of the field and defines geography as the study of man in relation to so much of his environment as varies locally; $\mathrm{Mr}$. Mackinder thus takes geography to be the physical basis of history. He insists on a clear separation being made between physical geography and both geology and physiography. The physical geographer has to deal with only so much of the past as will enable him to interpret the present, whereas the geologist deals with the present, only that he may be able to interpret the past. So with other departments of science, as meteorology ; from the new stand-point only so much of them is to be included as is pertinent to the geographical line of investigation. Mr. Mackinder illustrated his position by two sets of three maps-one of South-Eastern England, physical, geological, political ; and the other of India, shorving physical features in relation to rainfall and population. He attempted to show, on the basis of physical conditions, why, among other things, London should have become the metropolis of the Empire and why the three southeastern counties should have had their existing boundaries. With reference to India, again, he showed how geographical conditions determined that Delhi and Calcutta should have become the ancient and modern capitals of India. Geography, when studied in this way, Mr. Mackinder thinks, might become a bridge between the physical sciences on the one hand and classical and historical studies on the other. The lecture was illustrated by some very fine and instructive maps and lantern views.

\section{PHYSICAL NOTES}

THE inverse electromotive force of the voltaic arc has recently been investigated by Prof. C. R. Cross, of the Massachusetts Institute of Technology, and by Mr. W. E. Shepard. It appears that with currents varying from 3 to 10 amperes the inverse electromotive force is about 39 volts when the arc is silent, and about I5 volts when it is hissing; but both these values show a diminution as the currents employed are increased. The transition from one state to the other is abrupt. Addition of volatile metallic salts to the arc always decreases the inverse electromotive foree. In rarefied air the inverse electromotive force is unaltered, but the true re-istance of the arc is diminished.

Sensitive hygrometers have lately been constructed upon a principle resembling Bregnet's metallic thermometer. A spiral composed of two substances having different hygrometric coefficients of expansion tends to curl or uncurl according to changes in the hygrometric state of the air. Some of these have been made by Prof. W. Holtz out of thin brass spirals with a thin coating of gelatine on one side. Independently, M. Nodon, of Paris, has constructed some recording hygrometers having spirals made of Bristol board coated on one side with gelatine (with a little salicylic acid), and on the other with bitumen. The principle is not new : in various collections of physical apparatus similar arrangements have existed for at least a dozen years.

LENSES which magnify, and yet are perfectly flat on both sides, have been constructed by Schott and Co., of Jena, the manufacturers of Abbé's optical glass. These lenses are mere curiosities. They consist of single disks of glass, such that the refractive index decreases in a regular manner from the surface inwards. The properties of this arrangement have been investigated by Prof. K. Exner, of Vienna.

QUADRANT electrometers have been lately described by M. Ledeboer, in which the motion of the needle (often of very annoying duration) is damped so as to be aperiodic. This is achieved by making each of the four "quadrants" of steel highly magnetised. The needle is therefore damped by magnetic friction. The suspension is unifilar.

Several modifications have been lately introduced into the L.eclanché battery. Mr. Sydney Walker proposes to substitute sulphur for the manganese : it is cheaper, and less of it is required. Mr. A. Pollak does away with the manganese, but employs a special, porous, coarse, annular block of carbon, which stands half out of the liquid and absorbs oxygen from the air to depolarise. M. Germain introduces a novel material to hold the liquid, an absorbent preparation, chiefly cellulose, made from cocoa-nut fibre, and which has received the curious name of "cofferdam." It has truly remarkable absorbent properties, as it will suck up and hold from twelve to fourteen times its own weight of water. A "cofferdam" cell does not spill the liquid.

DRY portable cells appear to be coming into favour, gelatine being the favourite medium. They are claimed as novelties both in Paris and in Frankfort. Joule's "glue-battery"-a Daniell's cell, having a gelatinous mass impregnated with sulphates of copper and zinc-is the parent of all these later forms.

ELECTRIC welding is the latest of the industrial applications of electricity, and it would seem to have already reached a thoroughly practical stage. Prof. Elihu Thomson, of Lynn, Massachusetts, has shown that bars of iron, steel, copper, and brass can be welded firmly together in a few seconds by passing through their junction a very powerful electric current. He has invented a special kind of transformer or induction coil to enable him to accomplish this operation. It is possible thus to weld iron and brass together in a firm joint. Simultaneously, researches on the same subject have been made by two Russian gentlemen in the laboratory of M. Marcel Deprez in Paris, and they have announced their discovery under the name of "electrohephaest." If we are not mistaken, similar experiments were made before the Académie des Sciences some years ago by the late $M$. Rubmkorff, Moreover, in Mr. J. P. Joule's papers he mentions the discovery of the practicability of electric welding by himself and Sir William Thomson.

ACCORDING to Olszewski, the critical temperatures of nitrogen and oxygen are respectively $-1.46^{\circ},-118.8^{\circ}$, of the Centigrade scale.

$$
\text { ON THE MORPHOLOGY OF BIRDS } 1
$$

THERE are several things that go to increase the interest in the morphology of these culminating Sauropsida at the present time.

(I) The discovery by Gegenbaur, Huxley, and others, of the close relationship of birds and reptiles, especially of the extraordinary fact that the hind-limb and pelvis of even the most minute bird pass through a stage in which they correspond almost exactly with the hind-limb and pelvis of the most gigantic kinds of extinct reptiles-the Dinosaurs or Ornitho. scelida.

(2) The recent discoveries of biologists as to the composition of the cheiropterygium in the various types of air-breathing Vertebrata. It is now well known that the five-fingered hand and the foot with five toes are the specialised modern representatives of hands and feet that had at least seven rays in their composition.

And (3) the study of the development and general morphology of birds is at the present time of great interest, now that we are looking to the study of metamorphosis for some initial elucidation of the mystery as to the origin of the various types of Vertebrata.

The labour of each succeeding day at this culminating class makes it more and more impossible for me to conceive of birds as arising direct from the Dinosaurians, or indeed from any other order or group of reptiles.

Long attention to the metamorphosis of the Amphibia has intensified this difficulty to me; for the newly-transformed frog or newt appears to me to be the true counterpart of a newlyhatched reptile-snake, lizard, turtle, or crocodile.

Each of these young creatures, whether it has undergone a true metamorphosis or has been the subject of pre-natal transformation, is evidently an imago, although an imago that continues to grow.

Now each amphibian has its own larva, for the larvæ of the various species have their specific differences.

The thousand known species of existing Amphibia-Anurans, Urodeles, and Cæcilians-and all the fishes that undergo meta-

I Paper by Prof. W. K. Parker, F.R.S., read before the Royal Society on January 27,1887 . 
morphosis, are as truly, if not as remarkably, distinct from each other in their larval as in their imago form; as much so as is the case in insects, or any other of those invertebrate types that are truly metamorphic.

If many of the existing Vertebrata are metamorphic now, is it not very probable that they were all metamorphic once?

The fact that we have even now such forms as the larval lamprey (or Ammocœete), the larvæ of Ganoids and Dipnoi, and the tadpoles of newts and frogs, suggests to me the possibility of the existence of huge swarms of low proto.Vertebrata in the early ages of the inhabited planet.

If such proto-vertebrate forms existed, then it is quite supposable that a metamorphosis may, from time to time, have taken place, of this and that quasi-larval form into archaic reptile, ancestral bird, or primitive mammal.

I am not afraid that anyone familiar with the development, structure, and habits of the existing Amphibia will see any difficulty in the passage of a metamorphic into a so-called nonmetamorphic type, during time, and under the pressure of new outward conditions-when the dilemma offered to the supposed low vertebrate was Transform or perish.

To me it seems that the creature's necessity was Nature's opportunity; and that, during long ages, the morphological force had accumulated in those low forms an enormous surplusage of unused energy, which, in the ripeness of time, blosiomed out into this and that new and noble type.

Of all the types of Vertebrata, there is none like the bird of high degree for illustrating what Prof. Huxley calls "the threefold law of evolution," 1 namely, overgrowth of come parts, starvation and even death of others, and fusion of parts originally distinct.

No kind of vertebrate whatever presents to the os'eologist so hopeless an enigma in the adult skeleton, as that of the bird ; in the overgrowth of certain parts, the abortion or suppression of others, and the extensive fusion of large tracts of skeletal elements.

Hence this class has largely acted upon the morphological mind; the "comparative anatomist" has, of necessity, undergone evolution into the " morphologist," and the latter has had to be refined and developed into the "embryologist."

In the bird class we meet with this remarkable phenomenon, namely, that the swiftest creatures by far that inhabit the earth have had, for the purposes of their most consummate mechanism, the greatest loss of freedom of the individual parts of the skeletal framework.

Between the pigeon, on one hand, above, and the emu, on the other, below, there are several families of related birds ; but there is no direct superposition-they are obliquely above or below each other.

Amongst the Carinatie, which lie in the intermediate space, there is none better for the purposes of study than the common fowl ; to this type I have devoted most attention, and have now worked out the limbs in as many stages as I formerly did the skull.

I can now give an account of the vertebral column with the ribs and sternum, the limb-girdles and limbs, from the end of the seventh day of incubation; hy which time the hyaline cartilage is perfect, and certain even of the bony tracts are begun.

The fowl is an intermediate form between the emu and the pigeon, but most akin to the latter. I shall now confine myself to what is seen in the development of the skeleton (excluding the skuli) in this medium type.

The vertebral column, at the end of a week's incubation, is formed of hyaline cartilage; up to the end of the true sacrals, the notochord is completely invested with cartilage; but, behind those four segments, only at the sides.

The notochord has its constrictions in the middle of each centrum, and is most dilated at the intercentra.

The neural arches do not nearly meet above; the atlas is in four pieces - a superficial and an inner piece to the centrum, and a pair of arch-rudiments; the inner segment of the centrum becomes the odontoid process of the axis.

Between the axis and the first true sacral, all the vertebra have separate ribs; in the cervical region, except near the dorsal region, there are small styloid cartilages lying horizontally, which have their head, or thick end, wedged in between the upper and lower transverse processes. Near the dorsals they

I See his paper "On the Application of the Laws of Evolution to the Arrangement of the Vertebrata, and more especially of the Mammalia" (Zool. Soc. Proc., December $x_{4}, 188_{4}$, pp. 649.662 ). are transversely placed, and then begin to develop a descending process.

The first vertebra of this stage with complete ribs becomes, by absorption of the lower part of the arch, the last cervical in the adult. Behind the twenty pre-sacrals there are fifteen sacrals, and this series has its subdivisions.

The first develops ribs (it is dorso-sacral), the next three develop minute but distinct ribs, like those near the lower part of the neck; these are lumbo-sacral. Then come the four sacrals with no ribs, and then the seven uro-sacrals, the first two of which have rib-bars that ossify separately, below the upper transverse processes, which latter form a complete series from the third cervical to the last free caudal segment.

Of those there are five; then come five more paired imperfect rudiments, clinging to the terminal part of the notochord.

At the end of the eighth day there are six of these, with the last elongated, and the notochord projecting behind far enough for three or four more rudiments.

At the end of the tenth day the vertebral chain has undergone a great change. The atlas is still composed of four distinct pieces of cartilages, but the ribs have become fused above and below with the transverse processes, and the notochord is now most constricted at the intercentra.

Besides this, in the pre-sacrals, it is constricted in two places within each centrum; so that each centrum in the modern bird corresponds to three subdivisions of this axial chord.

For two or three days there is evidence of an archaic subdivision of the notochord into three times as many vertebral divisions as are made now in the modern bird.

In the sacral the constrictions are fewer; they are only at the intercentra, and in the middle of the centrum.

The only absolutely necessary part of the sternum is that where the sternal ribs are attached; that is a very small part, and the rest is for the attachment of the huge muscles that act upon the wings, and for the obliqui and recti abdominis.

The limb-girdles are each in three pairs of distinct cartilages. In front, the scapula, the minute pre-coracoid, the coracoid; behind, the ilium, pubis, and ischium; the pre-pubis is part of the ilium, and that has two regions, the pre-ilium and the postilium.

These parts in the bird are not continuous tracts of cartilages, ossified by several centres, but are distinct, first as cartilages, then as bony tracts ; those of the shoulder keep distinct; those of the hip soon coalesce.

The wings at the end of the seventh day are three-toed webbed paws, with all the digits turned inwards. The rods that compose the main part of it are composed of solid cartilage : the humerus, radius, ulna, and first and second metacarpals have a bony sheath round their middle part; the ends of the digits and the carpals are but partly chondrified. Five carpal nuclei, however, can be made out, and the two proximal nuclei are known to be further subdivided, each into two, in other types; hence we can already account for seven carpals in the bird, which has only two in the adult, in a free state.

Moreover, the first digit has two, and the second three phalanges, the normal number, as in lizards; the third, which should have four, but in birds has as a rule only one, has now two, as in the ostrich, and a few other birds; there is no sign at the end of the seventh or even of the eighth day of incubation of any more than three digits, but we have in the wrist an intermedio-radiale, a centralo-ulnare, and three distal carpals, answering to the three developed metacarpals. The digits up to the end of the eighth day are rounded and flattish, and are quite like those of a young newt or frog. But in two days more, at the end of the tenth day, the wing has almost acquired the adult form; and one more bony centre, that of the first metacarpal, has appeared. The overgrowth of the second distal carpal and the second metacarpal, with its large and dilated digit, has arrested the distal carpal of the first or short digit, the "pollex." This is the last nucleus to chondrify. It is still a very small, limpet-like disk of cartilage, and is now only to be seen on the flexor face of the manus, inside the top of the second metacarpal ; the distal carpal of the third ray is also small as compared with the large crescentic second distal nucleus. It is thrown Qn to the ulnar or outer side of the manus by the overgrowth of the middle rod and its carpal. The curve of the digits at their end is now, not inwards, or to the radial side, but outwards; and the two developed distal segments form now the core of two claws, that of the first, or pollex, being of considerable length. 
Thus, by the end of the tenth day, the reptilian type or fore foot has been attained, and the amphibian type lost, whilst the limb as a whole is now a fore-leg no longer, but a wing, thoroughly specialised by evolutional transformation.

The fore-limb has not simply become modified into a wing by the shortening of the pollex and third ray, the enlargement of the second, and the abortion of the fourth and fifth of a forepaw, like that of the lizard; but we have now the historical representatives of three more rays which have cropped up since the end of the eighth day.

I have repeatedly noticed that aborted parts, like overshadowed plants, are late to appear, and soon wither, or are arrested in their growth. This is the case here, for the new rays are late, small, and scarcely functional in the fullest development. They are not lost, however, but, like certain larval structures to be found in the skulls of the highest types of birds, they are built up into the finished wing, although they form an unimportant part of it as far as function goes.

The first of these additional rays is the "pre-pollex"; this is a lunate tract of fibro-cartilage attached to the inner face of the first metacarpal. The other two are composed of true hyaline cartilage, and appear, one on the ulnar side of the second, and the other on the ulnar side of the third developed metacarpal.

I have described them as intercalary metacarpals, for they seem to be the starved twins of the second and third large rays each distal carpal, very probably, in the archaic forms carried two rays. Thus there is supposed, for such a fore-limb, a digit inside the pollex of the modern bird, and then two pairs of rays, of which only the inner in each case has been retained.

The paddle of Ichthyosaurus shows this kind of primitive cheiropterygium admirably.

Thus we can account for seven carpals and six digits in the wing of the modern bird; in the legs the specialisation is not so intense, but is very great; the study of the embryonic stages shows in it many parts that the adult bird gives no signs of whatever.

Instead of there being even two tarsals, free and functional, there is only one, and that has merely the function of a "sesamoid," and has been mistaken continually for a bone of that sort, that nucleus answers to our naviculare, morphologically termed the "centrale."

Notwithstanding the extreme diversity in the habits of existing bird;, and the great difference seen in their shank bone, this part is always single, although composed of three metatarsals. As in reptiles, the joint at this part is not between the astragalus and tibia, as in mammals, but through the tarsal series ; no sign of this structure is seen in the adult bird. That which appears to be the condyloid end of the tibia is a row of tarsal bones, the tibiale, fibulare, and intermedium : these bave long been known as separate bones in young birds, but their distinctness in the early embryo as cartilaginous nuclei has only lately been made out.

I have been able, however, to demonstrate this repeatedly in different kinds of birds. The centrale also, although seen in the embryo as one of the tarsal series, was not properly identified; it is a constant element, but becomes degraded.

The distal series of tarsals exis's as a single tract of cartilage, and then as a single plate of bone. But it is related to three metatarsals, and the middle or thick part is the first to chondrify in the embryo, and to ossify in the chicken or young bird ; there are here three connate nuclei, with very slight signs of distinctness. The whole mass answers to our middle and external "cuneiform bones," and to the inner half of the "os marnum." Thus five tarsals can be always made out clearly, and two more accounted for.

The first metatarsal, which has been known, for some time, through the valuable researches of Morse, to have occasionally a proximal as well as a distal rudiment, has, I find, always a proximal rudiment as well.

Then, as Dr. G. Baur and Miss A. Johnson have shown, there is a fifth metatarsal ; it is a small pisiform cartilage, which so $n$ coalesces with the fourth, and with the great distal tarsal. I can only find a "pre-hallux" by turning to teratology, and this is not the lawful method.

There may, however, be some "reversion" or " atavism" in the polydactyle foot of the Dorking fowl, which has a welldeveloped "pre-hallux" and a double "hallux"; the twin digits of that part have $a$ very ichthyosaurian appearance.

\section{SCIENTIFIC SERIALS}

Annalen der Physik und Chemie, xxix., No. I1.-B. Dessau, on metal films arising from the disruption of a kathode. Discusses the production of mirror-like films such as obtained by Pliicker, Crookes, and Wright from the disgregation of the metal kathode in Geissler-tubes. With a pointed kathode and a flat glass surface as recipient, the film forms a flat conical deposit, showing interference-rings in reflected light, and proving also the presence of optical dispersive power in the metal. The dispersion in films of platinum, iron, nickel, and silver. It is anomalous in the case of gold and copper. The films are double-refracting, and, in the case of oxidisable metals, disappear on oxidation. -Ed. Hagenbach, propagation of electricity in telegraph-wires. Experiments made with chronographic apparatus on Swiss lines, together with a discussion of the results of Wheatstone, Walker, Guillemin, and others. Arguing from theory, the author compares, not the apparent speed, but the ratio of the time to the square of the length of circuit, $-\mathrm{B}$. von Kolenko, reply concerning the pyro-electricity of quartz. Maintains, against Prof. Hankel, that the poles of a warmed quartz crystal are not altered during cooling by passing through a flame. -E. Edlund, remarks on H. Hoppe's communication on the theory of unipolar induction.-S. von Wroblewski, on the representation of the rotation between the gaseous and liquid states of matter by isopyknal lines. The transition of state is represented by curves drawn on a diagram having, for given definite densities, pressures as ordinates and temperatures as abscissæ; such curves being termed isopykns or isopyknal lines. The result of examination of these curves shows that, though there is no such thing as an absolutely definite critical temperature or critical pressure per se, there is a critical density for every liquid. $-K$. Schmidt, on reflection at the surface of crystalline elliptically-polarising media. Experiments made with a crystal of cinnabar, and results compared with the formulæ of Voigt and that of Ketteler. The latter leads to closer correspondence than the former with the facts of observation.- $\mathrm{H}$. Muraoka, on the deformation of metal plates by grinding. The radius of the curvature produced by grinding metal disks set in a bed of fusible alloy is proportional to the cube of their thickness -K. Exner, validity of lens formulæ for non-homogeneous lenses.- E. Budde, a means of deciding between the electro-dynamic point-laws of Weber, Riemann, and Clausius. This gives the elementary theory of an experiment not yet made.-J. Kollert, on a new galvanometer. This is practically identical with Gray's form.

No. 12, 1886.-C. Fromme, on the galvanic polarisation evoked by small electromotive forces. This gives a first series of results with platinum electrodes in dilute sulphuric acid.Edm. Hoppe, on the theory of unipolar induction ; with a reply to Prof. Edlund.-F. Himstedt, on a determination of the quantity " $v$." The method was that of comparison of the two capacities of a condenser; the result $v=3^{\circ} 0074 \times 10^{10} \mathrm{~cm}$. $/ \mathrm{sec}$. -R. Lamprecht, on the action of the magnet upon electric discharges in rarefied gases, concludes that the law of Biot and Savart holds good as the calculated trajectories agree with curves observed by Hittorf in $1869 .-\mathrm{A}$. Foeppl, the spread of the electric charge in conductors. - L. Boltzmann, remarks on the opinion of Herr Lorberg on a point in electro-dynamics. W. Voigt, on the torsion of a rectangular prism of homogeneous crystalline substance; a mathematical investigation.-J. Kiewiet, on the elasticity of bending of pure zinc, copper, tin, and their alloys. The moduli of elasticity of alloys is not constant, but depends on the mode of preparation of the alloy as well as on its composition. Wertheim's rule for calculating the moduli of alloys from those of their components, according simply to the proportion of the constituents, appears to be inexact. The change of moduli of alloys with temperature is a simple linear function.-J. Stefan, on the relation between the theories of capillarity and evaporation.-A. Heritsch, on radiophony. The author combats Graham Bell's view that the condensed gases of a smoke deposit or carbon strip have something to do with its radiophonic properties. He finds that a coke plate heated to redness and then instantly placed in a tube and exposed to in termittent illumination from sunlight or electric light, emits tones. He further constructed a sort of flat glow-lamp, which, even when raised to brilliant incandescence, emitted tones when exposed to intermittent sunlight. No other source than sunlight was sufficient for this experiment.-G. Kobb, on the spectrum of germanium.-Greiner and Friedrichs, on a new mercury air- 